\title{
Numerical Taxonomy of Rapidly Growing, Scotochromogenic Mycobacteria of the Mycobacterium parafortuitum Complex: Mycobacterium austroafricanum sp. nov. and Mycobacterium diernhoferi sp. nov., nom. rev.
}

\author{
MICHIO TSUKAMURA, ${ }^{1 *}$ HERMINA J. VAN DER MEULEN, ${ }^{2}$ AND WILHELM O. K. GRABOW ${ }^{3}$
}

National Chubu Hospital, Obu, Aichi, Japan 474'; South African Institute for Medical Research, Johannesburg 2001, South Africa ${ }^{2}$; and National Institute for Water Research, Pretoria 0001, South Africa ${ }^{3}$

\begin{abstract}
A numerical analysis of phenetic data collected from rapidly growing, scotochromogenic mycobacterial strains isolated from water in South Africa and from strains of the taxa Mycobacterium parafortuitum, Mycobacterium aurum, Mycobacterium neoaurum, and "Mycobacterium diernhoferi" indicated that all of these organisms belong to the Mycobacterium parafortuitum complex. Our results also indicated that each of the taxa mentioned above is worthy of species status within the complex. The name Mycobacterium austroafricanum $\mathrm{sp}$. nov. is proposed for the South African isolates, and the characteristics of these isolates are described; the type strain is E9789-SA12441 (= ATCC 33464). The name Mycobacterium diernhoferi is revived for the organism described originally by Bönicke and Juhasz in 1965; the type strain of this species is strain 41001 (= ATCC 19340).
\end{abstract}

Three species of rapidly growing, scotochromogenic mycobacteria, Mycobacterium parafortuitum (16), Mycobacterium aurum (7), and Mycobacterium neoaurum (9), have not been clearly differentiated from each other in previous numerical classification schemes $(5,14)$ and have been considered members of a Mycobacterium parafortuitum complex. Saito et al. (5) showed that strains of "Mycobacterium diernhoferi" formed one cluster together with strains of $M$. parafortuitum. Based on this finding, " $M$. diernhoferi" was regarded as a synonym of $M$. parafortuitum. Recently, Kusunose et al. (M. Kusunose, E. Kusunose, I. Yano, S. Toriyama, H. Saito, and M. Tsukamura, Kekkaku 54:219$220,1979)$ found that $M$. parafortuitum contains 54- to 60-carbon mycolic acids, whereas " $M$. diernhoferi" contains 68- to 76-carbon mycolic acids. Furthermore, a number of strains of rapidly growing, scotochromogenic mycobacteria have been isolated from water in South Africa. These strains seem to represent a new taxon.

The purpose of the present study was to examine the taxonomic relationships among the three species of the $M$. parafortuitum complex, five strains of " $M$. diernhoferi," and a cluster of 23 South African strains isolated from water.

\section{MATERIALS AND METHODS}

Bacterial strains and cultivation techniques. All strains of $M$. parafortuitum, $M$. aurum, and $M$. neoaurum were isolated in the laboratory of the $\mathrm{Na}$ tional Chubu Hospital. These strains were maintained at $-20^{\circ} \mathrm{C}$ and were subcultured at 6-month intervals. Only the strains deposited in the American Type Culture Collection, Rockville, Md., are shown in Table 1. The type strains of all named species of rapidly growing, scotochromogenic mycobacteria were also included in this study (Table 1 ).

A total of 23 strains of rapidly growing, scotochromogenic mycobacteria were isolated from water in South Africa. These strains were isolated as follows. Samples of water were pretreated by adding $1.25 \%$ oxalic acid, which was neutralized after $10 \mathrm{~min}$ with $2 \%$ sodium hydroxide. After filtration, the filters were incubated at $37^{\circ} \mathrm{C}$ for 9 days on enriched Middlebrook $7 \mathrm{H} 9$ medium contáining cycloheximide (2). This medium contained $4.7 \mathrm{~g}$ of Middlebrook $7 \mathrm{H} 9$ broth base (BBL Microbiology Systems), $1 \mathrm{mg}$ of malachite green, $1 \mathrm{~g}$ of sodium propionate, $15 \mathrm{~g}$ of agar, $100 \mathrm{ml}$ of Middlebrook OADC enrichment (Difco Laboratories), $25 \mathrm{mg}$ of nalidixic acid, 25,000 $\mathrm{U}$ of penicillin, $50,000 \mathrm{U}$ of mycostatin, $100 \mathrm{mg}$ of cycloheximide, and $1,000 \mathrm{ml}$ of distilled water.

A total of 107 biochemical or physiological characters were studied for each strain. Of these, 104 were used in a previous study (15), with methods described previously $(10,12)$. The three additional characters used were growth at $42^{\circ} \mathrm{C}$, resistance to 5 -fluorouracil $(20 \mu \mathrm{g} / \mathrm{ml})$ in Ogawa egg medium, and resistance to mitomycin $\mathrm{C}(5 \mu \mathrm{g} / \mathrm{ml})$ in Ogawa egg medium. Growth at $42^{\circ} \mathrm{C}$ was assayed by incubating test strains at $42 \pm$ $0.5^{\circ} \mathrm{C}$ for 7 days.

Numerical analysis. Numerical classification was carried out by using a method described previously (15). Clustering was made by the single-linkage method (6). According to Orchard and Goodfellow (4), single- and average-linkage methods give the same clusters. 
In addition, a numerical analysis was also carried out to define the ranges of species (taxon), as follows. Ranges were measured by calculating $\mathrm{M} \pm 2 \mathrm{SD}$ or $\mathrm{M}$ $\pm 1.7 \mathrm{SD}$, where $\mathrm{M}$ is the mean matching coefficient (mean $M$ value) for individual strains of each species relative to a hypothetical median organism pattern (HMO) (3) within that species or another species and $\mathrm{SD}$ is the standard deviation. The range $\mathrm{M} \pm 2 \mathrm{SD}$ contains ca. $95 \%$ of the individual strains within a species (11). For example, if two species (taxa), species $\mathrm{A}$ and $\mathrm{B}$, are distinct, the range for species $\mathrm{A}$ relative to the $\mathrm{HMO}$ of species A should be higher than the range for species $B$ relative to the $\mathrm{HMO}$ of species A. There should be no overlap, or the overlap should occur in less than $5 \%$ of the strains within the proposed species. When we calculate $M-2 S D$ for species A relative to its own $\mathrm{HMO}$, a range higher than the lower limit of the range should contain ca. $97.5 \%$ of the individual strains in species A. On the other hand, when we calculate $M+2 S D$ for species $B$ relative to the HMO of species A, a range lower than the upper limit for the species should contain $97.5 \%$ of the strains in species B. Thus, if the lower limit of species $A$ is higher than the higher limit of species $B$, the two species are regarded as distinctly separate and have either no overlap or an overlap of less than $2.5 \%$ of the individual strains within the species. If the lower limit of species $A$ is measured as $M-1.7 \mathrm{SD}$, the higher limit of species $B$ is measured as $M+1.7 \mathrm{SD}$, and the former limit is higher than the latter limit, the two species are regarded as separate. Again, there should be no overlap, or an overlap of less than $5 \%$ of the individual strains within each species (8).

\section{RESULTS AND DISCUSSION}

The results of our numerical analysis are shown in Fig. 1 as a dendrogram. Five clusters were observed at a matching coefficient $(M$ value) level of $91 \%$, although a few intermediate strains were present between the clusters. The first cluster was composed of the strains isolated from water from South Africa, and the remaining four clusters contained the type strains of $M$. aurum, $M$. neoaurum, " $M$. diernhoferi," and $M$. parafortuitum. Therefore, the latter four clusters were considered to be a complex of four distinct species, and the five groups could be combined into one large cluster at a level of $90 \%$. Only one type strain of another species, Mycobacterium rhodesiae, was included in this large cluster.

The strains and species in the five clusters are shown in Table 1, whereas the biochemical and physiological properties of the groups are summarized in Table 2 . The $M$. parafortuitum cluster was composed of two subgroups; one contained the type strain of the species, and the other contained three strains named by Saito et al. (5) as Kanazawa strains.

The $M$. aurum and $M$. neoaurum clusters each contained a number of strains labeled as other species, and these two species have been frequently confused with each other in identification techniques. This situation was clarified by our study. The major distinguishing features of these two species are summarized in Table 3.

FIG. 1. Dendrogram showing relationships among strains studied. See Table 1 for strain serial numbers.
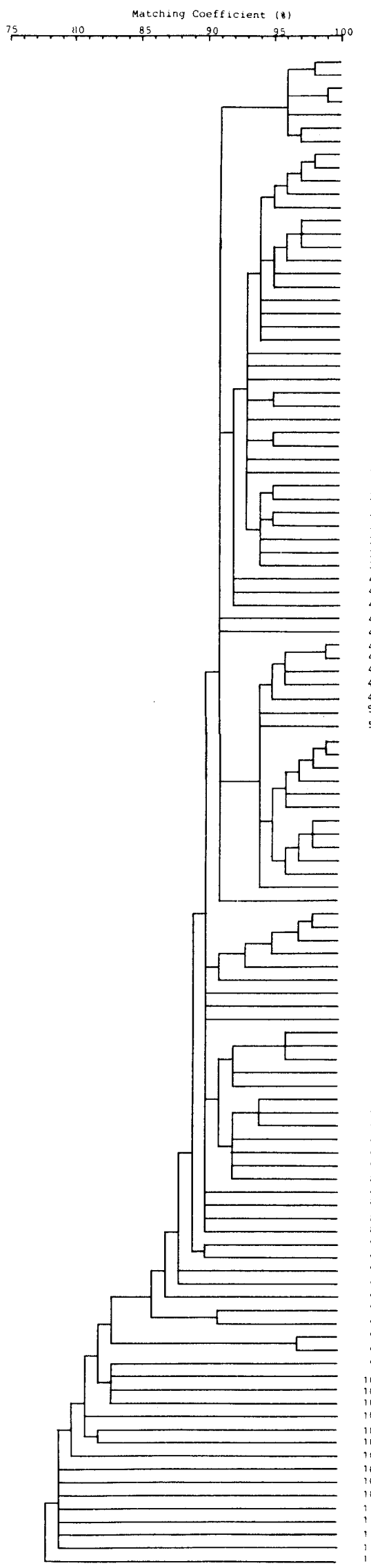
品 
TABLE 1. Mycobacterial strains used and cluster arrangement

\begin{tabular}{|c|c|c|c|c|c|}
\hline Cluster & $\begin{array}{l}\text { Serial } \\
\text { no. }\end{array}$ & $\begin{array}{l}\text { Laboratory } \\
\text { no. }^{a}\end{array}$ & Received as: & Source ${ }^{b}$ & Habitat $^{c}$ \\
\hline \multirow[t]{4}{*}{$\begin{array}{l}\text { M. austroafri- } \\
\text { canum }\end{array}$} & 1 & E9789B & $\begin{array}{l}\text { M. austroafricanum } \mathrm{SA} 2441^{\mathrm{T}} \\
\left(=\text { ATCC } 33464^{\mathrm{T}}\right)\end{array}$ & 1 & Water, South Africa \\
\hline & 2 & E9790B & $\begin{array}{l}\text { M. austroafricanum SA12442 } \\
(=\text { ATCC } 33465)\end{array}$ & 1 & Water, South Africa \\
\hline & 3 & E9789A & $\begin{array}{l}\text { M. austroafricanum SA12441 } \\
\left(=\text { ATCC } 33464^{\mathrm{T}}\right)\end{array}$ & 1 & Water, South Africa \\
\hline & 4 & E9790A & $\begin{array}{l}\text { M. austroafricanum SA12442 } \\
(=\text { ATCC } 33465)\end{array}$ & 1 & Water, South Africa \\
\hline \multirow[t]{11}{*}{ M. aurum } & 8 & 10005 & M. neoaurum ATCC 25799 & 2 & Soil, Japan \\
\hline & 9 & 10006 & M. neoaurum ATCC $25800^{d}$ & 2 & Soil, Japan \\
\hline & 19 & 10004 & M. neoaurum ATCC 25798 & 2 & Soil, Japan \\
\hline & 23 & 10001 & $M$. neoaurum ATCC 25791 & 2 & Soil, Japan \\
\hline & 31 & 15012 & $M$. aurum Lausanne 708 & 3 & Soil, Japan \\
\hline & 32 & 15061 & M. aurum ATCC 25792 & 2 & Soil, Japan \\
\hline & 34 & 15035 & $M$. aurum ATCC 25803 & 2 & Soil, Japan \\
\hline & 37 & 15013 & M. aurum ATCC 25793 & 2 & Soil, Japan \\
\hline & 40 & 15006 & $\begin{array}{l}\text { M. aurum ATCC } 23366^{\mathrm{T}} \\
\left(=\text { NCTC } 10437^{\mathrm{T}}\right)\end{array}$ & 2 & Soil, Japan \\
\hline & 41 & 15067 & M. aurum ATCC $25794^{e}$ & 2 & Soil, Japan \\
\hline & 44 & 15022 & M. aurum ATCC $27797^{e}$ & 2 & Soil, Japan \\
\hline \multirow[t]{5}{*}{ M. neoaurum } & 45 & 10003 & M. neoaurum ATCC 25796 & 2 & Soil, Japan \\
\hline & 47 & 10002 & $\begin{array}{l}M . \text { neoaurum ATCC } 25795^{\mathrm{T}} \\
\left(=\mathrm{NCTC} 10818^{\mathrm{T}}\right)\end{array}$ & 2 & Soil, Japan \\
\hline & 52 & 15001 & M. aurum NCTC 10439 & 4 & Soil, Japan \\
\hline & 53 & 15501 & $\begin{array}{l}M . \text { aurum ATCC } 25790 \\
(=\text { NCTC } 10440)\end{array}$ & 2 & Soil, Japan \\
\hline & 63 & 15509 & M. aurum ATCC 25801 & 2 & Soil, Japan \\
\hline \multirow{5}{*}{$\begin{array}{l}\text { "M. diernho- } \\
\text { feri" }\end{array}$} & 65 & 41001 & "M. diernhoferi" АТCC $19340^{\mathrm{T}}$ & 2 & Soil, Germany \\
\hline & 66 & 41003 & "M. diernhoferi" ATCC 19341 & 2 & Soil, Germany \\
\hline & 67 & 41004 & "M. diernhoferi" ATCC 19344 & 2 & Soil, Germany \\
\hline & 68 & 41006 & "M. diernhoferi" ATCC 25959 & 2 & Soil, Germany \\
\hline & 69 & 41005 & "M. diernhoferi" ATCC 25958 & 2 & Soil, Germany \\
\hline \multirow[t]{7}{*}{$\begin{array}{l}\text { M. parafor- } \\
\text { tuitum }\end{array}$} & 74 & 16001 & $\begin{array}{l}\text { M. parafortuitum ATCC } 19687 \\
(=\text { NCTC } 10410)\end{array}$ & 2 & Soil, Japan \\
\hline & 75 & 16002 & $\begin{array}{l}\text { M. parafortuitum ATCC } 19686^{\mathrm{T}} \\
\left(=\text { NCTC } 10411^{\mathrm{T}}\right)\end{array}$ & 2 & Soil, Japan \\
\hline & 76 & 16003 & M. parafortuitum ATCC 19688 & 2 & Soil, Japan \\
\hline & 82 & 16008 & M. parafortuitum ATCC $25808^{f}$ & 2 & Soil, Japan \\
\hline & 83 & 16010 & M. parafortuitum ATCC $25810^{f}$ & 2 & Soil, Japan \\
\hline & 84 & 16011 & $M$. parafortuitum ATCC $25811^{f}$ & 2 & Soil, Japan \\
\hline & 85 & 16033 & M. parafortuitum ATCC 25807 & 2 & Soil, Japan \\
\hline \multirow[t]{10}{*}{ Others } & 86 & 16007 & M. parafortuitum ${ }^{g}$ & 5 & Sputum, humans \\
\hline & 87 & 16041 & M. parafortuitum ${ }^{g}$ & 5 & Sputum, humans \\
\hline & 88 & 16044 & $M$. parafortuitum ${ }^{g}$ & 5 & Sputum, humans \\
\hline & 89 & 02002 & $M$. rhodesiae ATCC $27024^{\mathrm{T}}$ & 2 & Sputum, humans \\
\hline & 90 & 16009 & $M$. parafortuitum ${ }^{g}$ & & \\
\hline & 92 & 01039 & $\begin{array}{l}\text { Mycobacterium thermoresistibile } \\
\text { ATCC } 19527^{\mathrm{T}}\end{array}$ & 2 & Soil, Japan \\
\hline & 93 & 03001 & $\begin{array}{l}\text { Mycobacterium gadium ATCC } \\
27726^{\mathrm{T}}\end{array}$ & 6 & Sputum, humans \\
\hline & 94 & 21007 & $\begin{array}{l}\text { Mycobacterium vaccae ATCC } \\
15483^{\mathrm{T}}\end{array}$ & 2 & Soil, Germany \\
\hline & 95 & 48013 & $\begin{array}{l}\text { Mycobacterium chubuense } \\
\text { ATCC } 27278^{\mathrm{T}}\end{array}$ & 2 & Soil, Japan \\
\hline & 99 & 18112 & $\begin{array}{l}\text { Mycobacterium fortuitum ATCC } \\
6841^{\mathrm{T}}\end{array}$ & 2 & Skin lesion, human \\
\hline
\end{tabular}


TABLE 1-Continued

\begin{tabular}{|c|c|c|c|c|c|}
\hline Cluster & $\begin{array}{c}\text { Serial } \\
\text { no. }\end{array}$ & $\begin{array}{l}\text { Laboratory } \\
\text { no. }^{a}\end{array}$ & Received as: & Source ${ }^{b}$ & Habitat $^{c}$ \\
\hline & 100 & 22012 & $\begin{array}{l}\text { Mycobacterium cheloeni subsp. } \\
\text { abscessus ATCC } 19977^{\mathrm{T}}\end{array}$ & 2 & Gluteal abscess \\
\hline & 101 & 29505 & $\begin{array}{l}\text { Mycobacterium duvalii } \mathrm{NCTC} \\
358^{\mathrm{T}}\end{array}$ & 7 & $?$ \\
\hline & 102 & 33001 & $\begin{array}{l}\text { Mycobacterium flavescens ATCC } \\
14474^{\mathrm{T}}\end{array}$ & 2 & Guinea pig \\
\hline & 103 & 19054 & $\begin{array}{l}\text { Mycobacterium chelonei subsp. } \\
\text { chelonei } \text { NCTC } 946^{\mathrm{T}}\end{array}$ & 7 & $?$ \\
\hline & 104 & E8757 & $\begin{array}{l}\text { Mycobacterium komossense } \\
\text { ATCC } 33013^{\mathrm{T}}\end{array}$ & 8 & $\begin{array}{l}\text { Sphagnum vegeta- } \\
\text { tion }\end{array}$ \\
\hline & 105 & 49005 & $\begin{array}{l}\text { Mycobacterium aichiense ATCC } \\
27280^{\mathrm{T}}\end{array}$ & 2 & Soil, Japan \\
\hline & 106 & E8761 & $\begin{array}{l}\text { Mycobacterium sphagnii ATCC } \\
33027^{\mathrm{T}}\end{array}$ & 8 & $\begin{array}{l}\text { Sphagnum vegeta- } \\
\text { tion }\end{array}$ \\
\hline & 107 & 14025 & $\begin{array}{l}\text { Mycobacterium phlei ATCC } \\
11758^{\mathrm{T}}\end{array}$ & 2 & $?$ \\
\hline & 108 & 29006 & $\begin{array}{l}\text { Mycobacterium gilvum NCTC } \\
10742^{\mathrm{T}}\end{array}$ & 7 & $?$ \\
\hline & 109 & 47503 & $\begin{array}{l}\text { Mycobacterium tokaiense ATCC } \\
27282^{\mathrm{T}}\end{array}$ & 2 & Soil, Japan \\
\hline & 110 & 47001 & $\begin{array}{l}\text { Mycobacterium obuense ATCC } \\
27023^{\mathrm{T}}\end{array}$ & 2 & Sputum, humans \\
\hline & 111 & 90012 & Mycobacterium agri ATCC $27406^{\mathrm{T}}$ & 2 & Soil, Japan \\
\hline & 112 & 17027 & $\begin{array}{l}\text { Mycobacterium smegmatis } \\
\text { ATCC } 14468\end{array}$ & 2 & $?$ \\
\hline & 113 & 31002 & $\begin{array}{l}\text { Mycobacterium chitae ATCC } \\
19627^{\mathrm{T}}\end{array}$ & 2 & Soil, Japan \\
\hline & 114 & 18256 & $\begin{array}{l}\text { Mycobacterium snegalense } \\
\text { NCTC } 10956^{\mathrm{T}}\end{array}$ & 9 & $?$ \\
\hline
\end{tabular}

${ }^{a}$ The suffix A indicates before lyophilization and the suffix B indicates after lyophilization.

$b 1$, H. J. Nieuwoudt, South African Institute for Medical Research, Johannesburg, South Africa; 2, American Type Culture Collection, Rockville, Md.; 3, University of Lausanne, Lausanne, Switzerland; 4, National Collection of Type Cultures, London, England; 5, M. Tsukamura, National Chubu Hospital, Obu, Aichi, Japan; 6, M. Casal Roman, University of Cordaba, Cordaba, Spain; 7, J. L. Stanford, Middlesex Hospital, London, England; 8, J. Kazda, Forschungsinstitut Borstel, Borstel, Federal Republic of Germany; 9, M. P. Lechevalier, Rutgers University, New Brunswick, N.J.

c All 23 strains of $M$. austroafricanum were isolated in South Africa, and all strains of M. parafortuitum, $M$. aurum, and $M$. neoaurum were isolated in Japan.

${ }^{d} M$. parafortuitum according to Saito et al. (5).

e Outside the $M$. aurum cluster.

${ }^{f}$ Kanazawa strains according to Saito et al. (5).

${ }^{8}$ Outside the $M$. parafortuitum cluster.

In the study of Saito et al. (5) strains of " $M$. diernhoferi" formed a cluster together with strains of $M$. parafortuitum, and thus the former name was regarded as a synonym of $M$. parafortuitum. However, based on our study, we believe that these two organisms can be differentiated by a number of markers (Table 4 ).

In a previous study (14), in which 80 test characters were examined, three species $(M$. parafortuitum, $M$. aurum, and $M$. neoaurum) were somewhat difficult to differentiate from each other. Our results based on the 107 characters described here provide a more adequate differentiation of these three species. Although Saito et al. (5) used some additional markers (177 tests), their study contained a number of linked characters (since participants carried out various tests independently), and inclusion of linked characters is known to disturb the formation of clear-cut clusters (13). In our study, we omitted linked characters where possible, and we believe that our results show a better separation of the three mycobacterial clusters.

To confirm the distinctions described above, we performed a numerical analysis by using the ranges of species as measured by the expression $\mathrm{M} \pm 1.7 \mathrm{SD}$. Table 5 shows the results of a numerical analysis of the relationships among $M$. parafortuitum, M. aurum, and M. neoaurum. By estimating $\mathrm{M}$ values relative to the $\mathrm{HMO}$ of $M$. parafortuitum, $M$. neoaurum could be differentiated from $M$. parafortuitum (as the lower 
TABLE 2. Biological and physiological characteristics of strains examined

\begin{tabular}{|c|c|c|c|c|c|}
\hline \multirow[b]{2}{*}{ Character $^{a}$} & \multicolumn{5}{|c|}{$\%$ of strains showing positive reaction } \\
\hline & $\begin{array}{l}\text { M. austro- } \\
\text { africanum } \\
(n=23)\end{array}$ & $\begin{array}{c}\text { M. aurum } \\
(n=35)\end{array}$ & $\begin{array}{c}\text { M. neo- } \\
\text { aurum } \\
(n=19)\end{array}$ & $\begin{array}{c}\text { M. para- } \\
\text { fortuitum } \\
(n=12)\end{array}$ & $\begin{array}{l}\text { "M. diern- } \\
\text { hoferi" } \\
(n=5)\end{array}$ \\
\hline Strongly acid-fast & 100 & 100 & 95 & $100(100)^{b}$ & 100 \\
\hline Weakly or partially acid-fast & 100 & 100 & 100 & $100(100)$ & 100 \\
\hline Permanent mycelium & 0 & 0 & 0 & $\begin{array}{ll}0 & (0)\end{array}$ & 0 \\
\hline Temporary mycelium & 0 & 0 & 0 & $(0)$ & 0 \\
\hline Long rods $(>7 \mu \mathrm{m}$ long) & 0 & 0 & 0 & $0 \quad(0)$ & 20 \\
\hline Intermediate rods ( 3 to $6 \mu \mathrm{m}$ long) & 13 & 91 & 79 & $58 \quad(20)$ & 40 \\
\hline Short rods $(<2 \mu \mathrm{m}$ long $)$ & 100 & 86 & 89 & $100(100)$ & 100 \\
\hline Cross barring & 0 & 0 & 0 & $\begin{array}{ll}0 & (0)\end{array}$ & 0 \\
\hline Cord formation & 0 & 0 & 0 & $0 \quad(0)$ & 0 \\
\hline Rough colonies & 0 & 17 & 0 & $92(80)$ & 0 \\
\hline Pigmentation of colonies in the dark & 96 & 100 & 100 & $0 \quad(0)$ & 0 \\
\hline Photochromogenicity & 0 & 0 & 0 & $50(100)$ & 0 \\
\hline Growth at $28^{\circ} \mathrm{C}$ & 100 & 100 & 100 & $100(100)$ & 100 \\
\hline Growth at $37^{\circ} \mathrm{C}$ & 100 & 100 & 100 & $100(100)$ & 100 \\
\hline Growth at $42^{\circ} \mathrm{C}$ & 0 & 0 & 26 & $42(100)$ & 0 \\
\hline Growth at $45^{\circ} \mathrm{C}$ & 0 & 0 & 0 & $0 \quad(0)$ & 0 \\
\hline Growth at $52^{\circ} \mathrm{C}$ & 0 & 0 & 0 & $\begin{array}{ll}0 & (0)\end{array}$ & 0 \\
\hline Growth after 3 days & 100 & 100 & 100 & $100(100)$ & 100 \\
\hline Resistance to $0.2 \%$ p-aminosalicylate & 100 & 100 & 100 & $100(100)$ & 100 \\
\hline p-Aminosalicylate degradation & 0 & 0 & 0 & $\begin{array}{ll}0 & (0)\end{array}$ & 0 \\
\hline Resistance to $\mathrm{NH}_{2} \mathrm{OH} \cdot \mathrm{HCl}(125 \mu \mathrm{g} / \mathrm{ml})$ & 100 & 89 & 100 & $75(100)$ & 100 \\
\hline Resistance to $\mathrm{NH}_{2} \mathrm{OH} \cdot \mathrm{HCl}(250 \mu \mathrm{g} / \mathrm{ml})$ & 65 & 51 & 95 & $17(40)$ & 0 \\
\hline Resistance to $\mathrm{NH}_{2} \mathrm{OH} \cdot \mathrm{HCl}(500 \mu \mathrm{g} / \mathrm{ml})$ & 0 & 6 & 84 & $0 \quad(0)$ & 0 \\
\hline Growth on Sauton agar medium & 100 & 100 & 100 & $100(100)$ & 100 \\
\hline $\begin{array}{l}\text { Growth on Sauton agar containing } 0.1 \% \\
\text { sodium salicylate }\end{array}$ & 100 & 29 & 84 & $50(100)$ & 80 \\
\hline Degradation of salicylate & 0 & 0 & 0 & $\begin{array}{ll}0 & (0)\end{array}$ & 0 \\
\hline $\begin{array}{l}\text { Tolerance to } 0.1 \% \text { picric acid (Sauton } \\
\text { agar) }\end{array}$ & 100 & 100 & 100 & $100(100)$ & 100 \\
\hline $\begin{array}{l}\text { Tolerance to } 0.2 \% \text { picric acid (Sauton } \\
\text { agar) }\end{array}$ & 100 & 100 & 100 & $100(100)$ & 100 \\
\hline Arylsulfatase ( 3 days) & 100 & 14 & 89 & $25 \quad(40)$ & 0 \\
\hline Arylsulfatase (14 days) & 100 & 29 & 100 & $75(100)$ & 60 \\
\hline $\begin{array}{l}\text { Resistance to } 1 \mu \mathrm{g} \text { of thiophene-2-car- } \\
\text { boxylic acid hydrazide per ml }\end{array}$ & 100 & 100 & 100 & $100(100)$ & 100 \\
\hline Resistance to $0.05 \%$ sodium salicylate & 100 & 100 & 100 & $100(100)$ & 100 \\
\hline Resistance to ethambutol $(5 \mu \mathrm{g} / \mathrm{ml})$ & 0 & 3 & 100 & $0 \quad(0)$ & 0 \\
\hline Tolerance to $0.1 \% \mathrm{NaNO}_{2}$ (Sauton agar) & 100 & 100 & 100 & $100(100)$ & 100 \\
\hline Tolerance to $0.2 \% \mathrm{NaNO}_{2}$ (Sauton agar) & 96 & 83 & 100 & $83(100)$ & 100 \\
\hline $\begin{array}{l}\text { Growth on Sauton agar containing } 1 \% \\
\text { Tween } 80\end{array}$ & 100 & 100 & 100 & $100(100)$ & 100 \\
\hline $\begin{array}{l}\text { Resistance to } 0.5 \mathrm{mg} \text { of } p \text {-nitrobenzoic } \\
\text { acid per } \mathrm{ml}\end{array}$ & 100 & 97 & 100 & $58(100)$ & 100 \\
\hline Resistance to rifampin $(25 \mu \mathrm{g} / \mathrm{ml})$ & 100 & 89 & 100 & $50(100)$ & 100 \\
\hline Niacin production & 0 & 0 & 0 & $0 \quad(0)$ & 0 \\
\hline Tween hydrolysis after 7 days & 61 & 29 & 63 & $(0)$ & 0 \\
\hline Tween hydrolysis after 14 days & 61 & 40 & 63 & $\begin{array}{ll}0 & (0)\end{array}$ & 0 \\
\hline Catalase (foam height $>45 \mathrm{~mm}$ ) & 13 & 14 & 16 & $25 \quad(40)$ & 0 \\
\hline$\alpha$-Esterase & 39 & 14 & 11 & $8 \quad(0)$ & 0 \\
\hline$\beta$-Esterase & 100 & 94 & 100 & $50 \quad(80)$ & 100 \\
\hline$\beta$-Galactosidase & 0 & 9 & 5 & $42 \quad(60)$ & 0 \\
\hline Acid phosphatase & 0 & 0 & 0 & $0 \quad(0)$ & 60 \\
\hline Nitrate reduction after $6 \mathrm{~h}$ & 100 & 17 & 21 & $67 \quad(20)$ & 100 \\
\hline Nitrate reduction after $24 \mathrm{~h}$ & 100 & 17 & 74 & $67 \quad(20)$ & 100 \\
\hline Acetamidase & 0 & 71 & 100 & $50(100)$ & 100 \\
\hline Benzamidase & 0 & 0 & 16 & $\begin{array}{ll}0 & (0)\end{array}$ & 0 \\
\hline Urease & 100 & 97 & 100 & $100(100)$ & 100 \\
\hline Isonicotinamidase & 0 & 0 & 0 & $\begin{array}{ll}0 & (0)\end{array}$ & 0 \\
\hline Nicotinamidase & 100 & 74 & 100 & $100(100)$ & 100 \\
\hline
\end{tabular}


TABLE 2-Continued

\begin{tabular}{|c|c|c|c|c|c|}
\hline \multirow[b]{2}{*}{ Character $^{a}$} & \multicolumn{5}{|c|}{$\%$ of strains showing positive reaction } \\
\hline & $\begin{array}{l}\text { M. austro- } \\
\text { africanum } \\
(n=23)\end{array}$ & $\begin{array}{c}\text { M. aurum } \\
(n=35)\end{array}$ & $\begin{array}{c}M . \text { neo- } \\
\text { aurum } \\
(n=19)\end{array}$ & $\begin{array}{c}\text { M. para- } \\
\text { fortuitum } \\
(n=12)\end{array}$ & $\begin{array}{l}\text { "M. diern- } \\
\text { hoferi" } \\
(n=5)\end{array}$ \\
\hline Pyrazinamidase & 100 & 91 & 100 & $100(100)$ & 100 \\
\hline Salicylamidase & 0 & 0 & 0 & $0 \quad(0)$ & 0 \\
\hline Allantoinase & 0 & 71 & 100 & $17(20)$ & 80 \\
\hline Succinamidase & 0 & 0 & 0 & $0 \quad(0)$ & 0 \\
\hline Glutamate as $\mathrm{N}$ and $\mathrm{C}$ sources & 100 & 100 & 100 & $100(100)$ & 100 \\
\hline Serine as $\mathrm{N}$ and $\mathrm{C}$ sources & 0 & 0 & 0 & $\begin{array}{ll}0 & (0)\end{array}$ & 0 \\
\hline Glucosamine as $\mathrm{N}$ and $\mathrm{C}$ sources & 13 & 97 & 100 & $83(100)$ & 80 \\
\hline Acetamide as $\mathrm{N}$ and $\mathrm{C}$ sources & 4 & 74 & 100 & $0 \quad(0)$ & 0 \\
\hline Benzamide as $\mathrm{N}$ and $\mathrm{C}$ sources & 100 & 77 & 100 & (0) & 100 \\
\hline \multicolumn{6}{|l|}{ Monoethanolamine as $\mathrm{N}$ and $\mathrm{C}$ sources } \\
\hline $\begin{array}{l}\text { Trimethylene diamine as } \mathrm{N} \text { and } \mathrm{C} \\
\text { sources }\end{array}$ & 9 & 100 & 100 & $100(100)$ & 100 \\
\hline Glucose as $\mathrm{C}$ source (glutamate $\mathrm{N}$ ) & 100 & 100 & 100 & $100(100)$ & 100 \\
\hline Acetate as $\mathrm{C}$ source (glutamate $\mathrm{N}$ ) & 100 & 100 & 100 & $100(100)$ & 100 \\
\hline Succinate as $\mathrm{C}$ source (glutamate $\mathrm{N}$ ) & 100 & 100 & 100 & $100(100)$ & 100 \\
\hline Pyruvate as $\mathrm{C}$ source (glutamate $\mathrm{N}$ ) & 100 & 100 & 100 & $100(100)$ & 100 \\
\hline Acetate as $\mathrm{C}$ source & 100 & 100 & 100 & $100(100)$ & 100 \\
\hline Citrate as $\mathrm{C}$ source & 100 & 9 & 89 & $42(80)$ & 40 \\
\hline Succinate as $\mathrm{C}$ source & 100 & 100 & 100 & $58(100)$ & 20 \\
\hline Malate as $\mathrm{C}$ source & 100 & 100 & 100 & $58(100)$ & 0 \\
\hline Pyruvate as $\mathrm{C}$ source & 100 & 100 & 100 & $100(100)$ & 100 \\
\hline Benzoate as $\mathrm{C}$ source & 0 & 0 & 0 & $8 \quad(0)$ & 0 \\
\hline Malonate as $\mathrm{C}$ source & 0 & 3 & 26 & $8 \quad(0)$ & 0 \\
\hline Fumarate as $\mathrm{C}$ source & 100 & 100 & 100 & $50(100)$ & 0 \\
\hline Glucose as $\mathrm{C}$ source & 100 & 100 & 100 & $100(100)$ & 100 \\
\hline Fructose as $\mathrm{C}$ source & 100 & 100 & 100 & $100(100)$ & 100 \\
\hline Sucrose as $\mathrm{C}$ source & 100 & 17 & 5 & $58(100)$ & 0 \\
\hline Ethanol as $\mathrm{C}$ source & 100 & 91 & 100 & $92(100)$ & 100 \\
\hline$n$-Propanol as C source & 100 & 100 & 100 & $100(100)$ & 100 \\
\hline Propylene glycol as C source & 48 & 89 & 84 & $50(100)$ & 0 \\
\hline 1,3-Butylene glycol as $\mathrm{C}$ source & 100 & 43 & 68 & $0 \quad(0)$ & 0 \\
\hline 1,4-Butylene glycol as $C$ source & 0 & 3 & 0 & $0 \quad(0)$ & 0 \\
\hline 2,3-Butylene glycol as $\mathrm{C}$ source & 96 & 69 & 63 & $50 \quad(40)$ & 0 \\
\hline$n$-Butanol as C source & 100 & 86 & 100 & $92(80)$ & 100 \\
\hline Isobutanol as C source & 100 & 86 & 100 & $92(80)$ & 100 \\
\hline Acid from glucose & 100 & 54 & 100 & $0 \quad(0)$ & 100 \\
\hline Acid from mannose & 9 & 23 & 100 & $\begin{array}{ll}0 & (0)\end{array}$ & 100 \\
\hline Mannose as $\mathrm{C}$ source & 9 & 100 & 100 & $100(100)$ & 100 \\
\hline Galactose as $\mathrm{C}$ source & 0 & 20 & 42 & $\begin{array}{ll}0 & (0)\end{array}$ & 0 \\
\hline Arabinose as $\mathrm{C}$ source & 30 & 89 & 68 & $42 \quad(80)$ & 100 \\
\hline Xylose as $\mathrm{C}$ source & 100 & 97 & 68 & $42(80)$ & 100 \\
\hline Rhamnose as C source & 0 & 94 & 100 & $\begin{array}{ll}0 & (0)\end{array}$ & 0 \\
\hline Trehalose as C source & 0 & 46 & 100 & (0) & 0 \\
\hline Inositol as $\mathrm{C}$ source & 100 & 80 & 100 & $8 \quad(0)$ & 100 \\
\hline Mannitol as $\mathrm{C}$ source & 96 & 94 & 100 & $50(100)$ & 100 \\
\hline Sorbitol as C source & 0 & 11 & 0 & $8 \quad(0)$ & 0 \\
\hline Acetamide as $\mathrm{N}$ source & 100 & 91 & 100 & $92(100)$ & 100 \\
\hline Benzamide as $\mathrm{N}$ source & 0 & 0 & 84 & $8(20)$ & 0 \\
\hline Urea as $\mathbf{N}$ source & 100 & 74 & 100 & $92(100)$ & 100 \\
\hline Pyazinamide as $\mathbf{N}$ source & 100 & 54 & 100 & $92(100)$ & 100 \\
\hline Nicotinamide as $\mathrm{N}$ source & 100 & 40 & 100 & $92(100)$ & 60 \\
\hline Nitrate as $\mathbf{N}$ source & 100 & 49 & 100 & $92(100)$ & 80 \\
\hline Nitrite as $\mathrm{N}$ source & 0 & 3 & 89 & $50(20)$ & 0 \\
\hline Resistance to 5-fluorouracil $(20 \mu \mathrm{g} / \mathrm{ml})$ & 0 & 0 & 0 & $50(100)$ & 20 \\
\hline Resistance to mitomycin $(5 \mu \mathrm{g} / \mathrm{ml})$ & 0 & 0 & 0 & $0 \quad(0)$ & 0 \\
\hline
\end{tabular}

${ }^{a}$ Unless noted otherwise, resistance tests were performed in Ogawa egg medium, and tests for the utilization of carbohydrates were carried out by using ammoniacal nitrogen. Tests for the utilization of nitrogen sources were made by using glycerol as the carbon source.

${ }^{b}$ The values in parentheses indicate the percentages of strains showing positive reactions in the $M$. parafortuitum cluster at a $92 \%$ level $(M$. parafortuitum sensu stricto; $n=5)$. 
TABLE 3. Differentiation between strains of $M$. aurum and strains of $M$. neoaurum

\begin{tabular}{lcc}
\hline \multicolumn{1}{c}{ Character } & \multicolumn{2}{c}{$\begin{array}{c}\text { Of strains showing } \\
\text { positive reaction }\end{array}$} \\
\cline { 2 - 3 } & $\begin{array}{c}M . \text { aurum } \\
(n=35)\end{array}$ & $\begin{array}{c}M . \text { neoaurum } \\
(n=19)\end{array}$ \\
\hline Arylsulfatase (3 days) & 14 & 89 \\
Arylsulfatase (14 days) & 29 & 100 \\
Resistance to ethambutol & 3 & 100 \\
$\quad$ (5 $\mu$ g/ml) & & \\
Nitrate reduction (24 h) & 17 & 74 \\
Citrate as C source & 9 & 89 \\
Rhamnose as C source & 94 & 0 \\
Benzamide as N source & 0 & 84 \\
Nitrite as N source & 3 & 89 \\
\hline
\end{tabular}

TABLE 4. Differentiation between strains of $M$. parafortuitum and strains of " $M$. diernhoferi"

\begin{tabular}{lcc}
\hline \multicolumn{1}{c}{ Character } & $\begin{array}{c}\text { \% Of strains showing } \\
\text { positive reaction }\end{array}$ \\
\cline { 2 - 3 } & $\begin{array}{c}\text { M. parafor- "M. diern- } \\
\text { tuitum }\end{array}$ & $\begin{array}{c}\text { Moferi" } \\
\text { hof }\end{array}$ \\
\hline Photochromogenicity & 100 & 0 \\
Growth at $42^{\circ} \mathrm{C}$ & 100 & 0 \\
$\beta$-Galactosidase & 60 & 0 \\
Monoethanolamine as N and & 0 & 100 \\
$\quad$ C sources & 100 & 0 \\
Malate as C source & 100 & 0 \\
Fumarate as C source & 100 & 0 \\
Sucrose as C source & 100 & 0 \\
Propylene glycol as C source & 0 & 100 \\
Inositol as C source & 100 & 0 \\
Resistance to 5-fluorouracil & & \\
$(20 \mu \mathrm{g} / \mathrm{ml})$ & & \\
\hline
\end{tabular}

${ }^{a}$ Strains forming one cluster at a level of $92 \%$.

limit of the range for $\dot{M}$. parafortuitum was $81.0 \%$ and the higher limit of the range for $M$. neoaurum was $78.4 \%$ ). On the other hand, $M$. aurum could not be differentiated from $M$. parafortuitum, since there was a considerable overlap between the ranges for these two species. Conversely, when $M$ values relative to the HMO of $M$. aurum were analyzed, $M$. parafortuitum could be differentiated from $M$. aurum, whereas $M$. neoaurum was not clearly separated from $M$. aurum. Finally, when $\mathrm{M}$ values relative to the HMO of $M$. neoaurum were determined, both $M$. parafortuitum and $M$. aurum were differentiated from $M$. neoaurum. In view of the results described above, we believe that the three clusters can be regarded as three distinct species.

The type strain of $M$. rhodesiae was in the large cluster of strains in the $M$. parafortuitum complex (Fig. 1). The $M$ values of the $M$. rhodesiae type strain (ATCC 27024) relative to the HMOs of the organisms examined were as follows: $M$. parafortuitum, $78 \%$; $M$. aurum, 82\%; $M$. neoaurum, 81\%; " $M$. diernhoferi," $80 \%$; South African strains, $84 \%$. All of these $M$ values were outside the ranges estimated for the species described above (Tables 5 to 7) and our results confirm the previous finding (15) that indicated the distinct nature of $M$. rhodesiae.

The status of the South African isolates (Mycobacterium austroafricanum) was also examined by numerical analysis (Table 6). When we estimated the $\mathrm{M}$ values for individual strains of each species relative to their own HMOs and to the HMOs of four other groups, this group of strains isolated from water showed the highest mean $\mathrm{M}$ value and the smallest standard deviation, indicating that this group is very homogeneous. When we compared the $M$ values for these South African isolates with the HMOs of various species of the $M$. parafortuitum complex, the strains isolated from water were clearly differentiated from $M$. aurum and $M$. neoaurum, but they could not be separated from $M$. parafortuitum. In a reciprocal analysis (comparing $\mathrm{M}$ values of other groups with the HMO of the South African isolates), the South African isolates were distinct from all species of the $M$. parafortuitum complex. When we analyzed the situation with respect to strains of " $M$. diernhoferi," we found that this group is also distinct from other mycobacteria in the $M$. parafortuitum complex (Table 7).

In previous works, Saito et al. (5) and Tsukamura and Mizuno (14) did not believe that there was sufficient evidence to establish individual species for $M$. parafortuitum, $M$. aurum, and $M$.

TABLE 5. Numerical analysis of relationships among $M$. parafortuitum, $M$. aurum, and $M$. neoaurum

\begin{tabular}{|c|c|c|c|}
\hline \multirow{2}{*}{$\begin{array}{l}\text { HMO relative to } \\
\text { which } \mathrm{M} \text { values } \\
\text { were determined }\end{array}$} & \multicolumn{3}{|c|}{$\mathrm{M} \pm \mathrm{SD}(\%)^{a}$} \\
\hline & $\begin{array}{c}\text { M. parafor- } \\
\text { tuitum } \\
(n=12)\end{array}$ & $\begin{array}{c}M . \text { aurum } \\
(n=35)\end{array}$ & $\begin{array}{c}N . \text { neoaurum } \\
(n=19)\end{array}$ \\
\hline M. parafortuitum & $88.0 \pm 4.11$ & $79.1 \pm 4.50$ & $77.7 \pm 1.19$ \\
\hline M. aurum & $76.5 \pm 3.06$ & $90.8 \pm 4.57$ & $83.0 \pm 2.38$ \\
\hline M. neoaurum & $72.9 \pm 5.63$ & $82.3 \pm 3.70$ & $95.0 \pm 2.13$ \\
\hline
\end{tabular}

${ }^{a} \mathrm{M}$ is the mean $\mathrm{M}$ value for the individual strains of each species, and SD is the standard deviation. 
TABLE 6. Numerical analysis of relationships among South African strains isolated from water, " $M$. diernhoferi," $M$. parafortuitum, $M$. aurum, and $M$. neoaurum

\begin{tabular}{lccccc}
\hline $\begin{array}{c}\text { HMO relative to which M } \\
\text { values were determined }\end{array}$ & $\begin{array}{c}\text { South African } \\
\text { isolates } \\
(n=23)\end{array}$ & $\begin{array}{c}M . \text { parafortuitum } \\
(n=12)\end{array}$ & $\begin{array}{c}M . \text { aurum } \\
(n=35)\end{array}$ & $\begin{array}{c}\text { M. neoaurum } \\
(n=19)\end{array}$ & $\begin{array}{c}\text { "M. diernhoferi" } \\
(n=5)\end{array}$ \\
\hline South African isolates & $97.3 \pm 1.71$ & $76.4 \pm 3.40$ & $78.9 \pm 4.46$ & $82.1 \pm 2.41$ & $81.0 \pm 1.22$ \\
$M$. parafortuitum & $81.5 \pm 1.74$ & $88.0 \pm 4.11$ & & & \\
$M$. aurum & $80.2 \pm 1.72$ & & $90.8 \pm 4.57$ & $95.0 \pm 2.13$ & $97.2 \pm 1.79$ \\
$M$. neoaurum & $83.8 \pm 1.66$ & & & & \\
"M. diernhoferi" & $82.0 \pm 1.91$ & & & & \\
\hline
\end{tabular}

${ }^{a}$ See Table 5 , footnote $a$.

neoaurum, and so these three groups of organisms were regarded as parts of the $M$. parafortuitum complex. However, we believe that these three groups can be differentiated from each other and that they are sufficiently distinct to warrant status as Mycobacterium species. Our findings also confirm that there are a number of similarities among the individual species, so there is still some justification for retaining the designation $M$. parafortuitum complex. In addition, our results indicate that both " $M$. diernhoferi" and the South African strains isolated from water belong to the $M$. parafortuitum complex, although members of each of these two groups could be distinguished from named species within the complex. We believe that these two clusters of mycobacteria are also worthy of species status.

It is somewhat unusual that no common biochemical or physiological characteristic was observed among the five groups in the complex (Table 2). This finding differs from the situation observed in all other Mycobacterium complexes, including the slowly growing mycobacteria, the Mycobacterium tuberculosis complex, the Mycobacterium avium complex, and the Mycobacterium nonchromogenicum complex, where some common characters (12; unpublished data) are apparent.

In our study, the South African strains isolated from water were shown to be distinct from all named species of rapidly growing, scotochromogenic mycobacteria, and we propose that members of this group be named Mycobacterium austroafricanum $\mathrm{sp}$. nov. The basic biological and physiological characteristics of this species are summarized in Table 2. The following is a description of the species.

Mycobacterium austroafricanum (aus tro af ri ca num. of South Africa). Acid-fast, rod-shaped cells 2 to $6 \mu \mathrm{m}$ long and $0.5 \mu \mathrm{m}$ wide. Forms mucoid, yellowish pigmented colonies in the dark, showing stronger pigmentation after exposure to light. Grows within 3 days. Grows at 28 and $37^{\circ} \mathrm{C}$, but not at $42^{\circ} \mathrm{C}$. Resistant to $\mathrm{NH}_{2} \mathrm{OH} \cdot \mathrm{HCl}$ at a concentration of $125 \mu \mathrm{g} / \mathrm{ml}$, but susceptible to $\mathrm{NH}_{2} \mathrm{OH} \cdot \mathrm{HCl}$ at a concentration of $250 \mu \mathrm{g} / \mathrm{ml}$. Susceptible to isoniazid (10 $\mu \mathrm{g} / \mathrm{ml})$ and ethambutol $(5 \mu \mathrm{g} / \mathrm{ml})$; resistant to $5 \%$ $\mathrm{NaCl}, p$-nitrobenzoic acid $(0.5 \mathrm{mg} / \mathrm{ml})$, rifampin $(25 \mu \mathrm{g} / \mathrm{ml})$, and thiophene-2-carboxylic acid hydrozide $(1 \mu \mathrm{g} / \mathrm{ml})$. Grows on Sauton agar medium containing either $0.1 \%$ sodium salicylate, $0.1 \% \mathrm{NaNO}_{2}$, or $0.2 \%$ picric acid.

Niacin is not produced. Catalase activity (semiquantitative) negative, $\alpha$-esterase negative, $\beta$-esterase positive, $\beta$-galactosidase negative, acid phosphatase negative, Tween 80 hydrolysis (after 14 days) negative, and arylsulfatase (after 3 days) positive. Nitrate reduced to nitrite (after $6 \mathrm{~h}$ ). Nicotinamidase and pyrazinamidase positive, but negative for benzamidase, isonicotina-

TABLE 7. Numerical analysis of relationships among " $M$. diernhoferi," $M$. parafortuitum, $M$. aurum, $M$. neoaurum, and South African strains isolated from water

\begin{tabular}{lccccc}
\hline \multirow{2}{*}{$\begin{array}{c}\text { HMO relative to which M } \\
\text { values were determined }\end{array}$} & $\begin{array}{c}\text { M. diernhoferi" } \\
(n=5)\end{array}$ & $\begin{array}{c}M . \text { parafortuitum } \\
(n=12)\end{array}$ & $\begin{array}{c}M . \text { aurum } \\
(n=35)\end{array}$ & $\begin{array}{c}M . \text { neoaurum } \\
(n=19)\end{array}$ & $\begin{array}{c}\text { South African } \\
\text { isolates } \\
(n=23)\end{array}$ \\
\hline "M. diernhoferi" & $97.2 \pm 1.79$ & $80.9 \pm 2.27$ & $80.7 \pm 2.43$ & $80.5 \pm 1.71$ & $82.0 \pm 1.91$ \\
$M$. parafortuitum & $81.6 \pm 1.52$ & $88.0 \pm 4.11$ & & & \\
$M$. aurum & $85.8 \pm 1.30$ & & $90.8 \pm 4.57$ & & $95.0 \pm 2.13$ \\
$M$. neoaurum & $80.6 \pm 2.41$ & & & & $97.3 \pm 1.71$ \\
South African isolates & $81.0 \pm 1.73$ & & & & \\
\hline
\end{tabular}

\footnotetext{
${ }^{a}$ See Table 5 , footnote $a$.
} 
midase, salicylamidase, allantoinase, and succinamidase.

Glutamate and monoethanolamine are utilized as simultaneous nitrogen and carbon sources, but serine, glucosamine, acetamide, benzamide, and trimethylene diamine are not utilized as such sources; acetate, citrate, succinate, malate, pyruvate, or fumarate is utilized as a sole source of carbon in the presence of ammoniacal nitrogen, but benzoate and malonate are not utilized as carbon sources. The following carbohydrates are utilized as sole sources of carbon in the presence of ammoniacal nitrogen: glucose, fructose, sucrose, ethanol, $n$-propanol, $n$-butanol, isobutanol, 1,3-butylene glycol, 2,3-butylene glycol, and xylose. The following carbohydrates are not utilized as sole sources of carbon: propylene glycol, 1,4-butylene glycol, mannose, galactose, arabinose, rhamnose, trehalose, and sorbitol. Inositol and mannitol are usually utilized as sole sources of carbon.

Source: water from South Africa.

Type strain: E9789-SA12441 $(=$ ATCC 33464).

Previously, "M. diernhoferi" was regarded as a synonym of $M$. parafortuitum (3). However, in the present study, we established that organisms in this group could be differentiated from $M$. parafortuitum. Furthermore, Kusunose et al. (Kekkaku 54:219-220, 1979) recently reported that $M$. parafortuitum contains 54- to 60-carbon mycolic acids, whereas " $M$. diernhoferi" strains contain 68- to 76-carbon mycolic acids. In view of these findings, we believe that the name " $M$. diernhoferi" should be revived for the organisms described originally by Bönicke and Juhasz (1) in 1965. The species description is as follows.

Mycobacterium diernhoferi (diern hó fer i. of Diernhof, who originally isolated the organisms). Acid-fast, rod-shaped cells 2 to $6 \mu \mathrm{m}$ long and $0.5 \mu \mathrm{m}$ wide. Grows within 3 days. Forms white, smooth colonies. Non-photochromogenic. Grows at 28 and $37^{\circ} \mathrm{C}$, but does not grow at $42^{\circ} \mathrm{C}$. Resistant to $\mathrm{NH}_{2} \mathrm{OH} \cdot \mathrm{HCl}$ at a concentration of $125 \mu \mathrm{g} / \mathrm{ml}$, but susceptible to $\mathrm{NH}_{2} \mathrm{OH} \cdot \mathrm{HCl}$ at a concentration of $250 \mu \mathrm{g} / \mathrm{ml}$. Resistant to isoniazid $(10 \mu \mathrm{g} / \mathrm{ml}), 5 \% \mathrm{NaCl}$, thiophene-2-carboxylic acid hydrazide (1 $\mu \mathrm{g} / \mathrm{ml})$, rifampin $(25 \mu \mathrm{g} / \mathrm{ml})$, and $p$-nitrobenzoic acid $(0.5 \mathrm{mg} / \mathrm{ml})$; susceptible to ethambutol (5 $\mu \mathrm{g} / \mathrm{ml})$. Tolerant of $0.2 \%$ picric acid and $0.1 \%$ $\mathrm{NaNO}_{2}$ in Sauton agar medium. Tween 80 not hydrolyzed after 14 days. Catalase (semiquantitative) negative, $\alpha$-esterase negative, $\beta$-esterase positive, and $\beta$-galactosidase negative. Nitrate reduced to nitrite after $6 \mathrm{~h}$. Acetamidase, urease, nicotiamidase, pyrazinamidase, and allantoinase positive, but negative for benzamidase, isonicotinamidase, salicylamidase, and succina- midase. Niacin is not produced. Glutamate, glucosamine, monoethanolamine, and trimethylene diamine are utilized as simultaneous nitrogen and carbon sources, but serine, acetamide, and benzamide are not utilized as such sources; acetate and pyruvate are utilized as sole sources of carbon in the presence of ammoniacal nitrogen, but citrate, succinate, malate, benzoate, malonate, and fumarate are not utilized as such sources. Glucose, fructose, mannose, arabinose, xylose, inositol, and mannitol are utilized as sole sources of carbon, but sucrose, galactose, rhamnose, trehalose, and sorbitol are not utilized as such sources. Ethanol, $n$-propanol, $n$ butanol, and isobutanol are utilized as sole sources of carbon, but propylene glycol, and 1,3-, 1,4-2,3-butylene glycols are not utilized as such sources.

Source: soil in a cattle field.

Type strain: 41001 (= ATCC 19340).

\section{LITERATURE CITED}

1. Bönicke, R., and S. E. Juhasz. 1965. Mycobacterium diernhoferi n. sp., eine in der Umgebung des Rindes häufig vorkommende neue Mycobacterium-Species. Zentralbl. Bakteriol. Parasitenkd. Infektionskr. Hyg. Abt. 1 Orig. 197:292-294.

2. Grabow, W. O. K., J. S. Burger, and E. M. Nupen. 1980. Evaluation of acid-fast bacteria, Candida albicans, enteric viruses and conventional indicators for monitoring wastewater res mation systems. Prog. Water Technol. 12:803-817.

3. Liston, J., W. Wiebe, and R. R. Colwell. 1963. Quantitative approach to the study of bacterial species. J. Bacteriol. 85:1061-1070.

4. Orchard, V. A., and M. Goodfellow. 1980. Numerical classification of some named strains of Nocardia asteroides and related isolates from soil. J. Gen. Microbiol. 118:295-312

5. Saito, H, R. E. Gordon, I. Juhlin, W. Käppler, J. B. G. Kwapinski, C. McDurmont, S. R. Pattyn, E. H. Runyon, J. L. Stanford, I. Tarnok, H. Tasaka, M. Tsukamura, and J. Weiszfeiler. 1977. Cooperative numerical analysis of rapidly growing mycobacteria. The second report. Int. J. Syst. Bacteriol. 27:75-85.

6. Sokal, R. R., and P. H. A. Sneath. 1963. Principles of numerical taxonomy. W. H. Freeman, San Francisco.

7. Tsukamura, M. 1966. Adansonian classification of mycobacteria. J. Gen. Microbiol. 45:253-273.

8. Tsukamura, M. 1971. Some considerations of classification of mycobacteria. Definition of bacterial species by introduction of the concept of "hypothetical median or mean organisms."' Jpn. J. Tuberc. Chest Dis. 17:18-30.

9. Tsukamura, M. 1972. A new species of rapidly growing, scotochromogenic mycobacteria. Mycobacterium neoaurum. Med. Biol. (Tokyo) 85:229-233.

10. Tsukamura, M. 1975 . Identification of mycobacteria. The National Chubu Hospital, Obu, Aichi, Japan.

11. Tsukamura, M. 1976. An approach to numerical identification of bacterial species. J. Gen. Microbiol. 95:207-212.

12. Tsukamura, M. 1976. Numerical classification of slowly growing mycobacteria. Int. J. Syst, Bacteriol. 26:409-420.

13. Tsukamura, M. 1977. Extended numerical taxonomy study of Nocardia. Int. J. Syst. Bacteriol. 27:311-323.

14. Tsukamura, M., and S. Mizuno. 1977. Numerical analysis of relationships among rapidly growing, scotochromogenic mycobacteria. J. Gen. Microbiol. 98:511-517.

15. Tsukamura, M., S. Mizuno, and S. Tsukamura. 1981. 
Numerical analysis of rapidly growing, scotochromogenic mycobacteria, including Mycobacterium obuense sp. nov., nom. rev., Mycobacterium rhodesiae sp. nov., nom. rev., Mycobacterium aichiense sp. nov., nom. rev., Mycobacterium chubuense sp. nov., nom. rev., and $M y$ - cobacterium tokaiense sp. nov., nom. rev. Int. J. Syst. Bacteriol. 31:263-275.

16. Tsukamura, M., H. Toyama, and S. Mizuno. 1965. Mycobacterium parafortuitum, new species. Med. Biol. (Tokyo) 70:232-235. 